\title{
ERROR ASSESSMENT OF A WAVELENGTH TUNABLE Frequency Domain System FOR Noninvasive Tissue SPECTROScopy
}

\author{
Brian W. Pogue* and Michael S. Patterson \\ McMaster University and Hamilton Regional Cancer Centre, 699 Concession Street, Hamilton, \\ Ontario, Canada L8V 5C2 \\ (Paper JBO-043 received Nov. 3, 1995; revised manuscript received May 9, 1996; accepted for publication May 10, 1996)
}

\begin{abstract}
A system is described and evaluated for noninvasive measurement of the scattering and absorption coefficients of tissue in the 600 to $850-\mathrm{nm}$ range. An arc lamp/monochromator combination provides a tunable source which is intensity modulated at $135 \mathrm{MHz}$ by a Pockel's cell and coupled by fiber optics to the tissue surface. Diffusely reflected light is collected by optical fiber bundles at distances of 1 and $2 \mathrm{~cm}$ from the source and the intensity ratio and phase difference between these two signals are measured. These data are analyzed by means of a diffusion model of light to provide estimates of the scattering and absorption coefficients. The accuracy of these estimates is assessed by calculation of the influence of instrumental noise, tissue optical properties, and modulation frequency. Because the analysis is based on the assumption of a semi-infinite tissue geometry, systematic errors caused by idealized finite volumes (slabs, cylinders, and spheres) are also considered. The biomedical utility of the system is illustrated by noninvasive measurement of the uptake of an intravenously injected dye in rabbit leg muscle. () 1996 Society of Photo-Optical Instrumentation Engineers.
\end{abstract}

Keywords tissue optics; noninvasive spectroscopy; diffusion theory; frequency domain.

\section{INTRODUCTION}

In the red and near infrared region of the optical spectrum (roughly 600 to $1200 \mathrm{~nm}$ ), light can readily penetrate several centimeters of tissue due to low intrinsic absorption. ${ }^{1}$ With appropriate illumination and detection geometries, relatively large tissue volumes can therefore be sampled and quantitative estimates of the tissue absorption coefficient may allow calculation of the concentration of endogenous or exogenous chromophores. The former include hemoglobin and cytochromes and the latter photosensitizers used in photodynamic therapy. Because of intense light scattering in tissue, quantitative determination of the absorption coefficient is a challenging problem which requires the application of physical models of light propagation.

In recent years a number of strategies have been proposed based on spatially resolved, ${ }^{2-6}$ time domain, ${ }^{7-10}$ or frequency domain ${ }^{11-15}$ measurements of light that has been diffusely reflected from the tissue. Patterson ${ }^{16}$ has recently reviewed the advantages of each approach and pointed out that the optimum method depends on the demands of the specific problem. The system described in this paper uses a light source that is intensity modulated at $135 \mathrm{MHz}$ and coupled by fiber optics to the tissue surface. Light that is detected on the tissue surface some distance away is modulated at the same frequency, but reduced in intensity and delayed in

*Current affiliation: Massachusetts General Hospital, Harvard Medical School, Boston, MA 02114. Address all correspondence to Michael S. Patterson. E-mail: mike_patterson@hrcc.on.ca time. A model of light propagation based on diffusion theory can be used to estimate the scattering and absorption coefficients of the tissue from the attenuation and time delay (phase shift). This approach offers the following specific advantages:

1. The use of an arc lamp or monochromator source allows selection of wavelength over a broad range. Complete absorption and scattering spectra can be acquired from wavelength scans.

2. Measurements are made at only one or two points on the tissue surface.

3. No absolute measurements are required.

4. Frequency domain techniques can provide quantum noise-limited detection at reasonable cost.

Except for the tunable source, the approach is similar in principle to that described previously by Fantini et al. ${ }^{14}$ who used four sources and a single detector. The work described in this paper had three main goals:

1. Characterization of the instrument noise and calculation of its influence on the precision of estimates of the tissue absorption and scattering coefficients. As well, a comparison was made between calculations based on measurements at one and two detector locations.

2. Determination of the influence of finite tissue volume on the estimates of the tissue optical

1083-3668/96/\$6.00 @ 1996 SPIE 
properties. Data reduction is usually based on the assumption that the tissue is a semiinfinite medium and previously we studied the systematic errors introduced by this assumption for a one-detector system. ${ }^{17}$ Here that work is extended to a system in which measurements are made at two detector locations.

3. Application of the system to a real biological problem: noninvasive measurement of the average tissue concentration of a dye similar to those used for photodynamic therapy. We believe this to be the first time frequency domain methods have been used to acquire complete quantitative in vivo absorption and scattering spectra.

\section{THEORY}

We assume that the biological tissue of interest is a macroscopically uniform turbid medium characterized by an optical absorption coefficient, $\mu_{\mathrm{a}}$, a transport (or effective isotropic) scattering coefficient, $\mu_{\mathrm{s}}^{\prime}$, and an average speed of light $c_{\mathrm{n}}$. In the wavelength region 600 to $1200 \mathrm{~nm}, \mu_{\mathrm{s}}^{\prime} \gg \mu_{\mathrm{a}}$, and diffusion theory has been shown to provide an accurate description of light propagation. ${ }^{18}$ The diffusion equation can be solved analytically for simple geometries and detailed results have been presented by Moulton ${ }^{19}$ and Arridge, Cope and Delpy. ${ }^{20}$ In this paper we are primarily interested in the simple geometry shown in Figure 1 where light delivery and detection occur on the surface of an "infinite" tissue half-space. ${ }^{21,22}$ As shown in an earlier paper, ${ }^{17}$ application of the extrapolated boundary condition leads to an expression for the AC component of reflectance, $R\left(\rho, \omega, \mu_{\mathrm{a}}, \mu_{\mathrm{s}}^{\prime}, c_{\mathrm{n}}\right)$, which is the number of photons crossing the tissue boundary per unit time per unit area at distance $\rho$ from a unit pencil beam source modulated at frequency $f$ $=\omega / 2 \pi$ given by

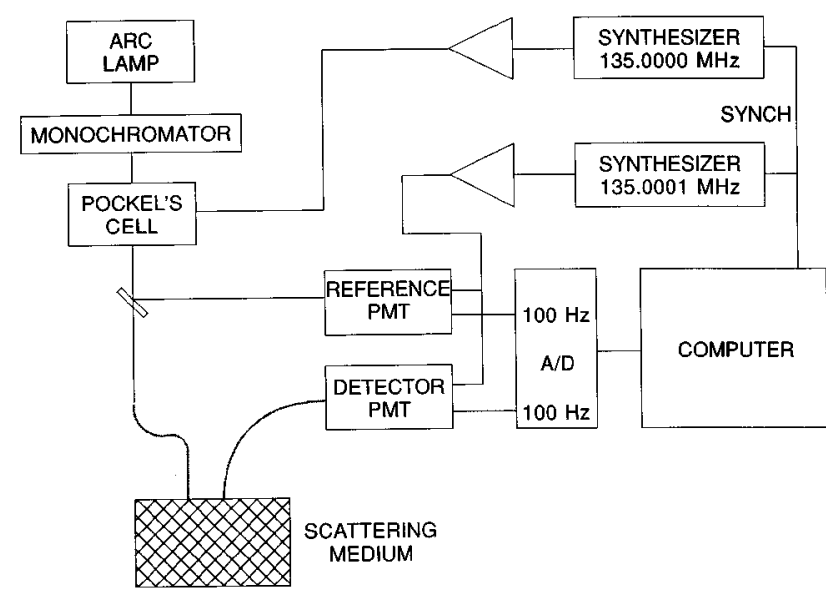

Fig. 1 Schematic diagram of the experimental apparatus for frequency domain measurements.

$$
\begin{aligned}
R\left(\rho, \omega, \mu_{\mathrm{a}}, \mu_{\mathrm{s}}^{\prime}, c_{\mathrm{n}}\right)= & \frac{1}{2(2 \pi)^{1 / 2}}\left[\frac{z_{\mathrm{o}}\left(1+k \rho_{\mathrm{o}}\right)}{\rho_{\sigma}^{3}} \exp \left(-k \rho_{\mathrm{o}}\right)\right. \\
& \left.+\frac{z_{p}\left(1+k \rho_{p}\right)}{\rho_{p}^{3}} \exp \left(-k \rho_{p}\right)\right], \quad \text { (1) }
\end{aligned}
$$

where $z_{\mathrm{o}}=1 / \mu_{\mathrm{s}}^{\prime}, z_{\mathrm{p}}=z_{\mathrm{o}}+4 D / \kappa, D=\left[3\left(\mu_{\mathrm{a}}\right.\right.$ $\left.\left.+\mu_{\mathrm{s}}^{\prime}\right)\right]^{-1}$ and $\kappa$ is a constant that depends on $c_{\mathrm{n}}$ and the speed of light in the nonscattering medium above the tissue. For a tissue-air boundary, $\kappa \simeq 0.426,{ }^{3}$ and for a Plexiglas-tissue boundary, $\kappa \simeq 1$.0. We also define $\rho_{\mathrm{o}}{ }^{2}=\rho^{2}+z_{\mathrm{o}}{ }^{2}, \rho_{p}{ }^{2}=\rho^{2}+z_{p}{ }^{2}$ and the complex attenuation coefficient:

$$
k=\left(\frac{\mu_{\mathrm{a}} c_{\mathrm{n}}+i \omega}{D c_{\mathrm{n}}}\right)^{1 / 2} .
$$

Note that $R\left(\rho, \omega, \mu_{\mathrm{a}}, \mu_{\mathrm{s}}^{\prime}, c_{\mathrm{n}}\right)$ is complex, so that the phase of the AC reflectance signal relative to the source $\theta$ is given by

$$
\theta=\arctan \frac{\operatorname{Im}[R]}{\operatorname{Re}[R]}
$$

and the amplitude $A$ is

$$
A=\left[\operatorname{Re}^{2}(R)+\operatorname{Im}^{2}(R)\right]^{1 / 2} .
$$

Of course it is not possible to have a source with negative intensity, so there is always a DC component present as well. This component is evaluated by setting $\omega=0$ in Eq. (1). The modulation is defined as the ratio of the AC to DC amplitudes

$$
M=A(\omega) / A(0),
$$

but for application of Eq. (1), the measured modulation $M_{\text {sig }}$ must be normalized by the source modulation, $M_{\text {src }}$, to yield

$$
M=M_{\text {sig }} / M_{\text {src }} \text {. }
$$

The goal of the measurements is to determine $\mu_{\mathrm{a}}$ and $\mu_{\mathrm{s}}^{\prime}$ from $R$. (It is usual to assume that $c_{\mathrm{n}} \cong 0.214 \mathrm{~m} \mathrm{~ns}^{-1}$ for soft tissue. ${ }^{23}$ ) Two general strategies have been adopted. One is to position a single detector at distance $\rho$ and to measure $M$ and $\theta .{ }^{11}$ The two equations (3) and (5) can be solved numerically for $\mu_{\mathrm{a}}$ and $\mu_{\mathrm{s}}^{\prime}$ once $M$ and $\theta$ are measured. This approach requires an additional reference measurement at $\rho=0$, which is usually accomplished by abutting the source and detector fiber bundles. An alternative method is to measure the phase and DC intensity at two distances $\rho_{1}$ and $\rho_{2} \cdot{ }^{24}$ From Eq. (1) equations can be derived for $\theta\left(\rho_{2}\right)-\theta\left(\rho_{1}\right)$ and $A\left(\rho_{2}, 0\right) / A\left(\rho_{1}, 0\right)$ in terms of $\mu_{\mathrm{a}}$ and $\mu_{\mathrm{s}}^{\prime}$. These can be solved numerically for $\mu_{\mathrm{a}}$ and $\mu_{\mathrm{s}}^{\prime}$ and no reference measurement for $\rho=0$ is required. This approach can be extended to more than two detector positions (or, equivalently, one detector and multiple sources), in which case the estimation of $\mu_{\mathrm{a}}$ and $\mu_{\mathrm{s}}^{\prime}$ becomes an optimization problem. $^{14}$

The equations described above were derived for the semi-infinite geometry. In an earlier paper ${ }^{17}$ we assessed the influence of finite tissue size on esti- 
mates of $\mu_{\mathrm{a}}$ and $\mu_{\mathrm{s}}^{\prime}$ made with a one-detector phase and modulation system. In that study, analytical solutions of the diffusion equation for slab, cylinder, and sphere geometries were used to generate data which were then used as input to the algorithm based on semi-infinite geometry. Here we extend that study to a two-detector system where the observables are the phase difference $\theta\left(\rho_{2}\right)-\theta\left(\rho_{1}\right)$ and the intensity ratio $A\left(\rho_{2}, 0\right) / A\left(\rho_{1}, 0\right)$.

\section{APPARATUS AND METHODS}

The apparatus used for the measurement of phase and intensity of multiply scattered light is illustrated schematically in Fig. 1. The instrumentation is essentially an adaptation of the frequency domain fluorometer described by Gratton and Limkeman. ${ }^{25}$ The light source is a $300-\mathrm{W}$ xenon arc lamp with an integral parabolic reflector that produces a collimated beam. This beam is focused on the entrance slit of a monochromator, and the light that emerges from the exit slit is recollimated. The intensity of this beam is modulated by a Pockel's cell at $135 \mathrm{MHz}$, passes through a beam splitter (92\% transmission, $8 \%$ reflection), and is then focused onto a 3-mm diameter optical fiber bundle. This bundle delivers the modulated light to the tissue surface. The DC intensity at the end of the fiber bundle is typically $10 \mu \mathrm{W}$ in a bandpass of $10 \mathrm{~nm}$, although there are much more intense peaks above $800 \mathrm{~nm}$. The modulation attained with the Pockel's cell is only about $8 \%$. Much higher modulation and intensity can be achieved with lasers or modulated LEDs, but none of these sources offers the wide tuning range of the arc lamp/monochromator combination.

Another 3-mm bundle collects scattered light at the tissue surface and its output is coupled via a lens to the photocathode of a photomultiplier tube (R928 Hamamatsu). A filter wheel can be used to interpose a bandpass filter between the bundle and the PMT. This may be necessary to reject fluorescent light under some circumstances, e.g., absorption spectroscopy of a fluorescent dye. A second PMT is also coupled via a fiber bundle to the light reflected from the beam splitter. This detector provides the reference for phase and intensity measurements. The photomultiplier tubes were always operated with $1000 \mathrm{~V}$ from anode to cathode and the source was attenuated when necessary.

Both PMTs are mounted in special housings (ISS, Champaign, Illinois) that allow the PMT gain to be modulated at high frequency by injection of an RF signal at the second dynode. If the gain modulation is performed at a frequency slightly offset (in this case, $100 \mathrm{~Hz}$ ) from the Pockel's cell modulation frequency, a $100 \mathrm{~Hz}$ heterodyne signal is generated which contains all the phase and amplitude information. This low frequency signal is sampled at 1 $\mathrm{kHz}$ and converted to a 12-bit digital value by a proprietary signal processing board (ISS, Cham- paign, Illinois). The data are acquired over a variable sampling period and then a fast Fourier transform is performed. The transform yields the DC amplitude, the AC amplitude, and the phase at the heterodyne frequency.

In vivo experiments were performed on three New Zealand white rabbits. Each rabbit was anesthetized by injection into the ear vein of $5 \mathrm{mg} / \mathrm{kg}$ xylazine, $50 \mathrm{mg} / \mathrm{kg}$ ketamine, and $1 \mathrm{mg} / \mathrm{kg}$ acepromazine maleate. The leg was shaved and a piece of black Plexiglas $0.6 \times 4 \times 6 \mathrm{~cm}$ was clamped over the femoral biceps muscle. Sufficient pressure was applied to ensure close contact between plastic and tissue. Three holes were drilled in the plastic: one held the source fiber bundle, the second held the detector bundle at a center-to-center distance of 1 $\mathrm{cm}$, and the third positioned the detector at $2 \mathrm{~cm}$ from the source. This arrangement, which minimized motion of the fibers relative to the muscle, was essential for reliable measurements. Data were acquired sequentially at the two source detector distances by manually moving the detector fiber bundle as required.

Data were acquired at 13 wavelengths from 560 to $830 \mathrm{~nm}$. The wavelength was then fixed at 660 $\mathrm{nm}$ and aliquots of the dye nickel phthalocyanine tetrasulfonate $\left(\mathrm{NiPcS}_{4}\right.$, Mid-Century Pharmaceuticals, Posen, Illinois) were injected via the ear vein. $\mathrm{NiPcS}_{4}$ has a strong absorption peak at $660 \mathrm{~nm}$, a weaker peak at $600 \mathrm{~nm}$, and no detectable fluorescence. Two solutions of $\mathrm{NiPcS}_{4}$ were used: 10 $\mathrm{mg} / \mathrm{ml}$ for the first two animals and $20 \mathrm{mg} / \mathrm{ml}$ for the third. After the injection of each $0.5 \mathrm{ml}$ aliquot, the signal at $1 \mathrm{~cm}$ source-detector separation was recorded for $15 \mathrm{~s}$. This was repeated three times to estimate the variance in the measurements, and then the detector fiber was moved to the second position. Four $15 \mathrm{~s}$ measurements were then performed at this distance. After injection of the last aliquot, measurements were again performed over the complete wavelength range. The in vivo results were analyzed by assuming matched boundary conditions, whereas the other results in this paper were derived for an air-tissue boundary.

\section{RESUlTS AND DISCUSSION}

This section of the paper is divided into four parts. First we demonstrate that for typical signal levels the system is quantum noise limited. Next we examine how noise in the measurements of phase and intensity affects the precision of the estimates of $\mu_{\mathrm{a}}$ and $\mu_{\mathrm{s}}^{\prime}$ made with one- and two-detector systems. The third part deals with systematic errors in $\mu_{\mathrm{a}}$ and $\mu_{\mathrm{s}}^{\prime}$ caused by finite tissue volumes for a two-detector instrument. Finally we present the results of in vivo measurements of $\mu_{\mathrm{a}}$ and $\mu_{\mathrm{s}}^{\prime}$ made with our two-detector system. 


\subsection{SYSTEM NOISE MEASUREMENTS}

To measure the typical variance in phase and amplitude, the source and detector fibers were fixed on the surface of a block of white linear polyethylene $(10 \times 10 \times 5 \mathrm{~cm})$. The detected signal was sampled for 30 consecutive 0.5 -s time intervals. The DC amplitude, AC amplitude and phase were calculated for each interval, as were the mean and standard deviation in each quantity. The source intensity was then reduced using a continuously variable neutral density filter (Newport Corp., Irvine, California) and the 30 measurements were repeated. In Figure 2 the standard deviations in the phase, AC amplitude, and DC amplitude are plotted as functions of the DC signal amplitude (in millivolts).

Also shown on these plots are the expected values of the standard deviation if the only source of noise is quantum fluctuations in the number of electrons emitted from the photocathode. In order to make this theoretical estimate, the source wavelength was set to $630 \mathrm{~nm}$ and the total optical power at the PMT end of the detector fiber was measured using a calibrated meter (Model 840, Newport Corp., Irvine, California). This meter can detect only a relatively high power, so the measured power of $1.2 \mu \mathrm{W}$ was further attenuated to a level of $0.12 \mathrm{nW}$, using calibrated neutral density filters before the photocathode. The PMT manufacturer states that the photocathode quantum efficiency at this wavelength is 0.07 , so that the photocathode current can be calculated by assuming that all of the light emitted by the detector bundle reaches the photocathode. Using these data and assumptions, we calculated that a DC signal of $1 \mathrm{~V}$ at $630 \mathrm{~nm}$ corresponds to a photocathode current of $2.7 \times 10^{6}$ electrons $\mathrm{s}^{-1}$. As explained in detail by Carlson, ${ }^{26}$ the standard deviation in the DC and AC signal amplitudes as well as the phase can then be calculated from knowledge of this average current and the sampling period. For example, in $0.5 \mathrm{~s}$, the total number of photoelectrons is, on average, $1.35 \times 10^{6}$ so the standard deviation would be the square root of this number, $1.16 \times 10^{3}$, and the predicted standard deviation in the $1-\mathrm{V}$ DC signal would be $0.86 \mathrm{mV}$. The standard deviation in the DC and AC amplitude would vary with the square root of the DC signal amplitude, and the standard deviation in phase would vary inversely as the square root of the DC signal amplitude, assuming that the modulation is constant. Similarly, the standard deviation in all three observables would ideally scale inversely with the square root of the total sampling time.

While other sources of noise may affect a tissue spectroscopy system, quantum fluctuations in the detected light impose an ultimate limit on performance. As shown in Figure 2, the system described here operates close to that theoretical limit under typical conditions. The propagation of these experi-
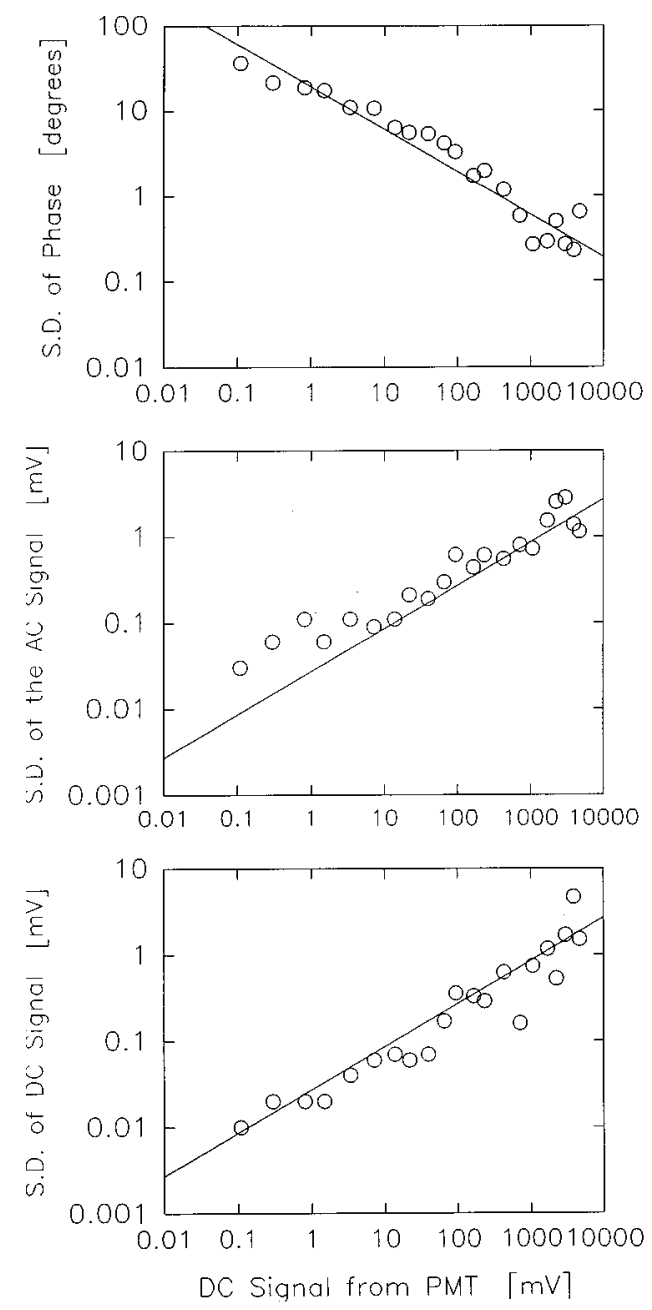

Fig. 2 Standard deviation in phase, AC signal, and DC signal as functions of the DC signal level. The points are experimental results for 0.5-s data acquisition, and the straight lines are theoretical predictions based on measurements of the incident optical power on the photocathode and the manufacturer's data for photocathode quantum efficiency. The modulation was $8 \%$.

mental uncertainties into the estimates of the tissue optical properties depends strongly on the optical properties themselves and the strategy used to deduce them.

\subsection{ERROR PROPAGATION OF RANDOM NOISE}

Since Eq. (1) does not have an analytical solution for $\mu_{\mathrm{a}}, \mu_{\mathrm{s}}^{\prime}$, it is necessary to perform a numerical Monte Carlo simulation to assess the propagation of experimental uncertainties into the estimates of the optical properties. We first considered a system with one detector located $2 \mathrm{~cm}$ from the source. Our practical experience with tissue in vivo has shown that this is the maximum distance at which useful signals can be obtained with the arc lamp source in the 600 to $850-\mathrm{nm}$ spectral range. First, Eq. (1) was used to calculate the phase and modulation of the detected signal (assuming 8\% source modulation) 
at $135 \mathrm{MHz}$ for given values of $\mu_{\mathrm{a}}$ and $\mu_{\mathrm{s}}^{\prime}$. We assumed that enough light was available to produce a full-scale DC signal $(4 \mathrm{~V})$, and values of the DC amplitude, AC amplitude, and phase were sampled from normal distributions having standard deviations corresponding to the theoretical data in Figure 2. (Since a 1-s integration time was assumed in this analysis, the data in Figure 2 were modified by dividing by the square root of 2.) An iterative Newton Raphson algorithm was then used to solve for $\mu_{\mathrm{a}}$ and $\mu_{\mathrm{s}}^{\prime}$ given the observed phase and modulation. This was repeated 200 times so that the standard deviation in $\mu_{\mathrm{a}}$ and $\mu_{\mathrm{s}}^{\prime}$ could be calculated, and required about $60 \mathrm{~s}$ of computation time for each set of coefficients. This process was repeated for 384 sets of optical properties where $\mu_{\mathrm{a}}$ was varied from
0.0025 to $0.06 \mathrm{~mm}^{-1}$ and $\mu_{\mathrm{s}}^{\prime}$ from 0.25 to 4.0 $\mathrm{mm}^{-1}$. The results are presented as lines of constant relative uncertainty as functions of $\mu_{\mathrm{a}}$ and $\mu_{\mathrm{s}}^{\prime}$; the uncertainty in $\mu_{\mathrm{a}}$ is shown in Figure 3(a) and the uncertainty in $\mu_{\mathrm{s}}^{\prime}$ in Figure 3(b). A value of, for example, 10, means that the standard deviation in the estimate of the interaction coefficient is equal to $10 \%$ of its true value.

The data in Figure 3 show that a system based on phase and modulation measurements at a single detector position gives $<10 \%$ uncertainty in $\mu_{\mathrm{a}}$ only when the absorption coefficient is less than $0.005 \mathrm{~mm}^{-1}$ and the scattering coefficient is greater than $1.0 \mathrm{~mm}^{-1}$. Performance degrades rapidly outside this regime, especially when $\mu_{\mathrm{a}}$ increases,
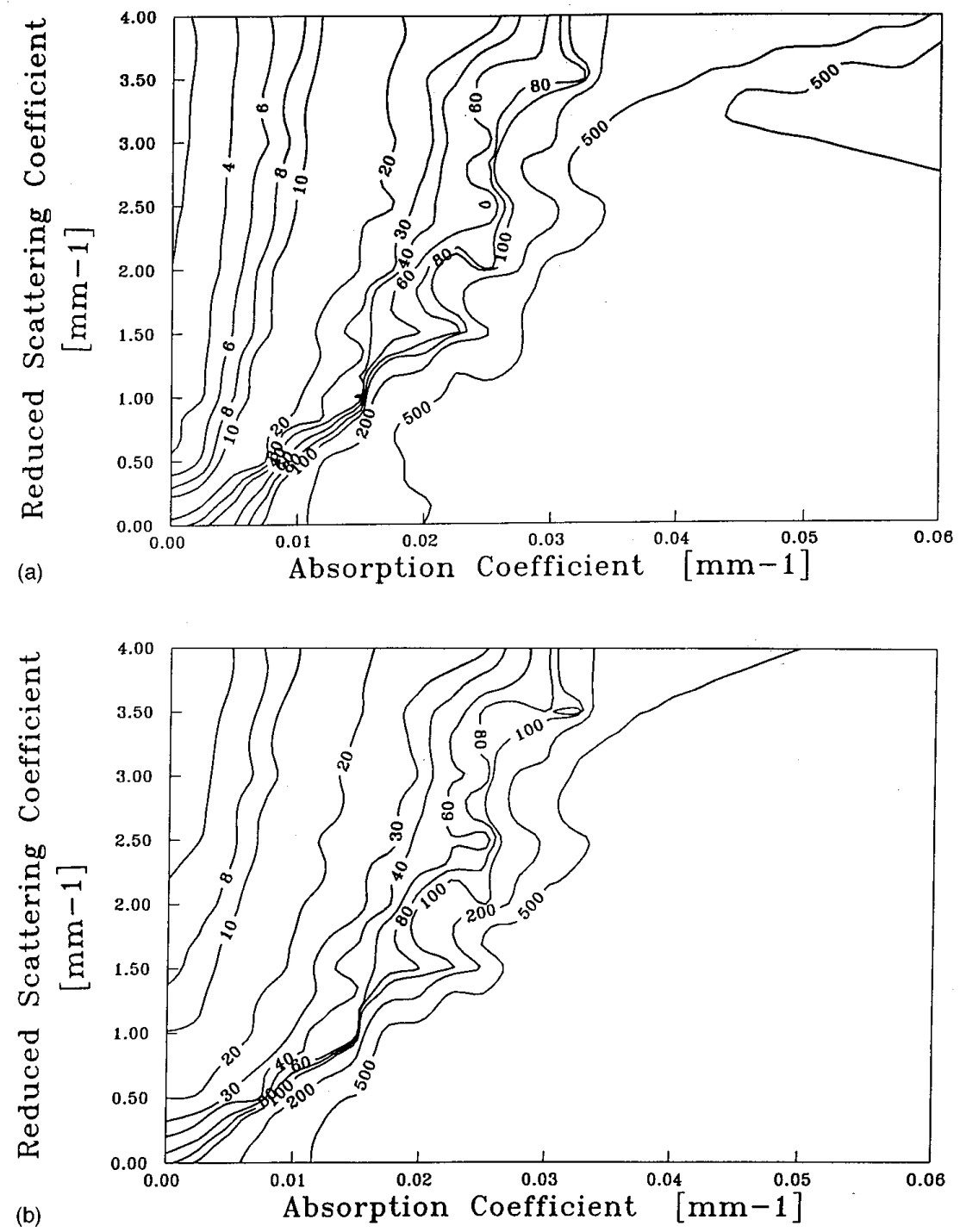

Fig. 3 Calculated percent relative uncertainty (standard deviation/value *100) in the absorption (a) and reduced scattering (b) coefficients as functions of the tissue optical properties for a system based on measurements of phase and modulation at a source-detector distance of $2 \mathrm{~cm}$ and $135 \mathrm{MHz}$-operation. Calculations were based on a 1-s data acquisition, theoretical shot-noise such as shown in Fig. 2, and numerical solution of Eq. (1). 
mainly because there is little demodulation and the uncertainty in this quantity dominates. This could be improved by operating at a higher frequency or higher modulation but, as mentioned earlier, this was not practical with our arc lamp system.

A more useful strategy is to employ two detectors at distances of 1 and $2 \mathrm{~cm}$. In this case the reflectance at the near detector will be greater than at the far detector. We assumed that sufficient light was available so that a full-scale signal was measured on the near detector. Equation (1) was then used to calculate the phase difference and intensity ratio, and standard deviations were assigned from the theoretical data in Figure 2. As described above, a Monte Carlo simulation was performed to deter- mine the uncertainties in $\mu_{\mathrm{a}}$ and $\mu_{\mathrm{s}}^{\prime}$ obtained by numerical solution of the equations derived from Eq. (1). The relative uncertainties in $\mu_{\mathrm{a}}$ and $\mu_{\mathrm{s}}^{\prime}$ are plotted for a range of tissue optical properties in Figs. 4(a) and 4(b), respectively.

Comparison of Figure 4(a) with Figure 3(a) shows that the regime over which $10 \%$ accuracy in $\mu_{\mathrm{a}}$ is achieved is comparable for the two approaches but that the two-detector method does not fail catastrophically as $\mu_{\mathrm{a}}$ increases. In this case the lower signal at the far detector is the dominant source of error, and better performance could be obtained if light reaching the near detector were attenuated by a well-calibrated filter. To simulate this approach,
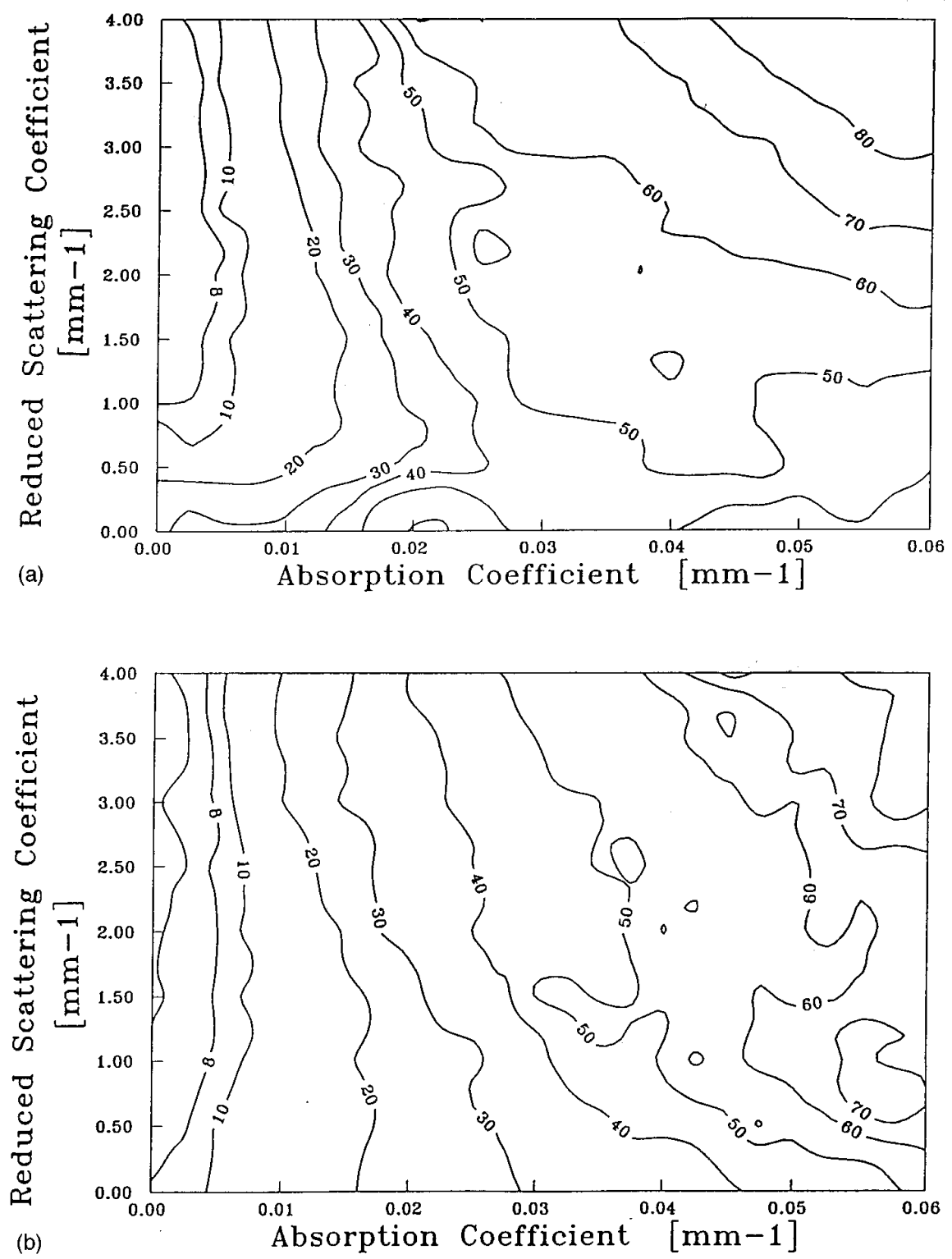

Fig. 4 Calculated percent relative uncertainty in absorption (a) and reduced scattering (b) coefficients for a system based on measurements of phase difference and intensity ratio between source-detector distances of 1 and $2 \mathrm{~cm}$. It was assumed that a full-scale signal was measured by the near detector and the signal at the far detector, calculated by Eq. (1), was smaller. 
we assumed that an attenuator was selected which resulted in equal signals at both detectors when $\mu_{\mathrm{s}}^{\prime}=1.0 \mathrm{~mm}^{-1}$ and $\mu_{\mathrm{a}}=0.01 \mathrm{~mm}^{-1}$. [The optical density of this filter would be 1.42 according to Eq. (1).] Clearly the signals could be equalized at any predetermined values of $\mu_{\mathrm{a}}$ and $\mu_{\mathrm{s}}^{\prime}$, but these were chosen as representative of tissues in the red and near infrared. ${ }^{1}$ The uncertainty in $\mu_{\mathrm{a}}$ which could be achieved with this equalized system is shown in Figure 5. The uncertainties in $\mu_{\mathrm{s}}^{\prime}$ (not shown) were comparable to those in $\mu_{\mathrm{a}}$. Comparison with Figure 4 (a) shows that $10 \%$ precision in the absorption coefficient can be achieved over a much wider range of optical properties $\left(\mu_{\mathrm{s}}^{\prime}>0.5 \mathrm{~mm}^{-1}\right.$ and $\mu_{\mathrm{a}}<0.02 \mathrm{~mm}^{-1}$ ).

The precision in $\mu_{\mathrm{a}}$ at $\mu_{\mathrm{a}}=0.01 \mathrm{~mm}^{-1}, \mu_{\mathrm{s}}^{\prime}=1.0$ $\mathrm{mm}^{-1}$, where the signals at both detectors were equalized, is about $4 \%$. It should be stressed that this is not an absolute limit and that it applies only to a system with the source characteristics described above. In principle, better performance can be achieved by use of a more intense source, a higher modulation frequency, greater modulation depth, or a longer measurement period. While it is not practical to do this with our arc lamp system, diode lasers can readily be modulated at higher frequencies with modulation approaching 100\%. Greater modulation is desirable because the rms phase noise is inversely proportional to the amplitude of the AC signal. Increasing the modulation frequency is useful because the relative error in the phase is inversely proportional to frequency. The effect of changing modulation frequency from 135 $\mathrm{MHz}$ to $500 \mathrm{MHz}$ on the uncertainty in $\mu_{\mathrm{a}}$ can be assessed by comparing Figure 6 with Figure 5. At best, the uncertainty is reduced to about $2 \%$. The effect of increasing the modulation from 8 to $100 \%$, while maintaining generation at $135 \mathrm{MHz}$, is shown by comparing Figure 7 with Figure 5 . The results in Figure 7 are comparable to those reported by Fantini et al. ${ }^{14}$ for their fixed wavelength LEDs modulated at $120 \mathrm{MHz}$.

\subsection{ERRORS DUE TO FINITE TISSUE VOLUME}

In this section we discuss the errors that occur when data obtained from a finite scattering medium are analyzed with the equation derived for an infinite half space, i.e., Eq. (1). We restricted our study to a two-detector system based on phase and DC intensity measurements at 1 and $2 \mathrm{~cm}$, because the performance of a one-detector system was assessed in a previous paper. ${ }^{17}$ The modulation frequency was $135 \mathrm{MHz}$. First we considered the case where the source and both detectors were located on the surface of an infinite slab of variable thickness. The analytical solution for the slab ${ }^{17}$ was used to calculate the reflectance at each detector and hence the phase difference and DC intensity ratio. These data were then used as the input to the iterative algorithm based on Eq. (1). Of course, if the tissue were a slab of known thickness, it would be possible to recover $\mu_{\mathrm{a}}$ and $\mu_{\mathrm{s}}^{\prime}$ exactly, but we are attempting to estimate the error inherent in applying the simplest model to an organ of unknown dimensions. No noise was added to the data in this case. Figure 8 shows the absolute error in the scattering and absorption coefficients as functions of slab thickness for slabs with $\mu_{\mathrm{s}}^{\prime}=1.0 \mathrm{~mm}^{-1}$ and

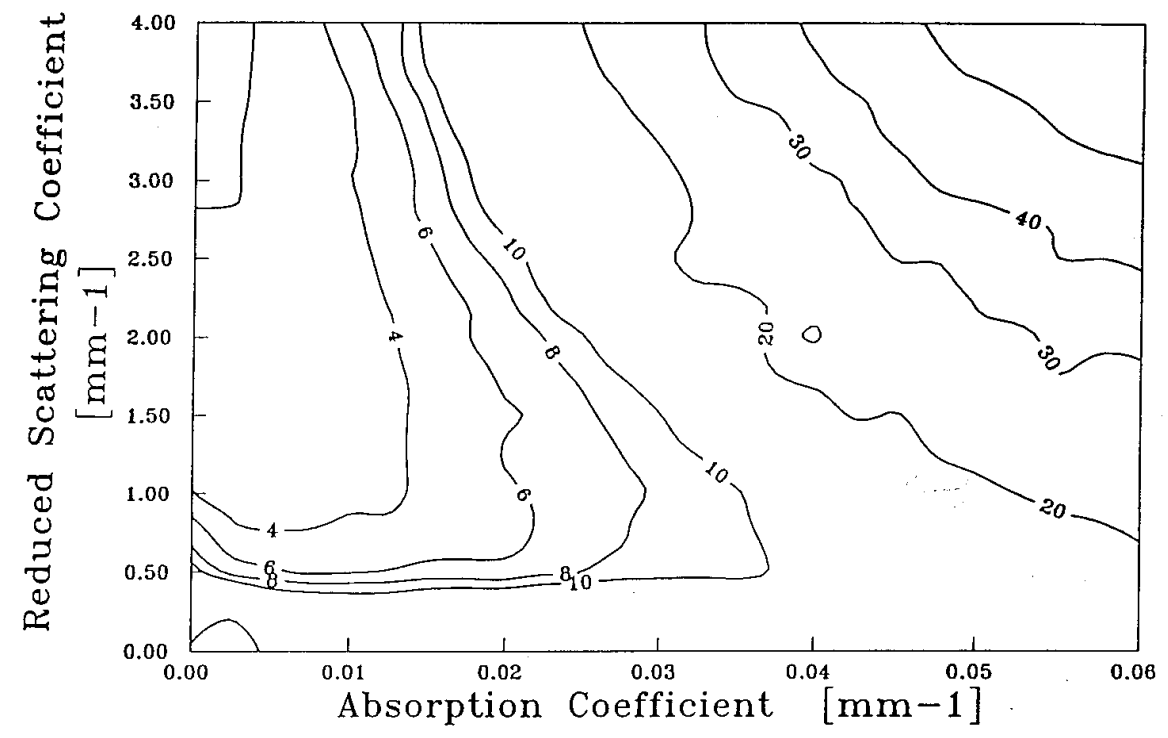

Fig. 5 Calculated percent relative uncertainty in the absorption coefficient for the same conditions as Fig. 4(a), except that an attenuator has been added to the detector at a $1-\mathrm{cm}$ distance so that the signal is equal to that measured at $2 \mathrm{~cm}$ for $\mu_{\mathrm{a}}=0.01 \mathrm{~mm}^{-1}$ and $\mu_{\mathrm{s}}^{\prime}=1.0 \mathrm{~mm}^{-1}$. 


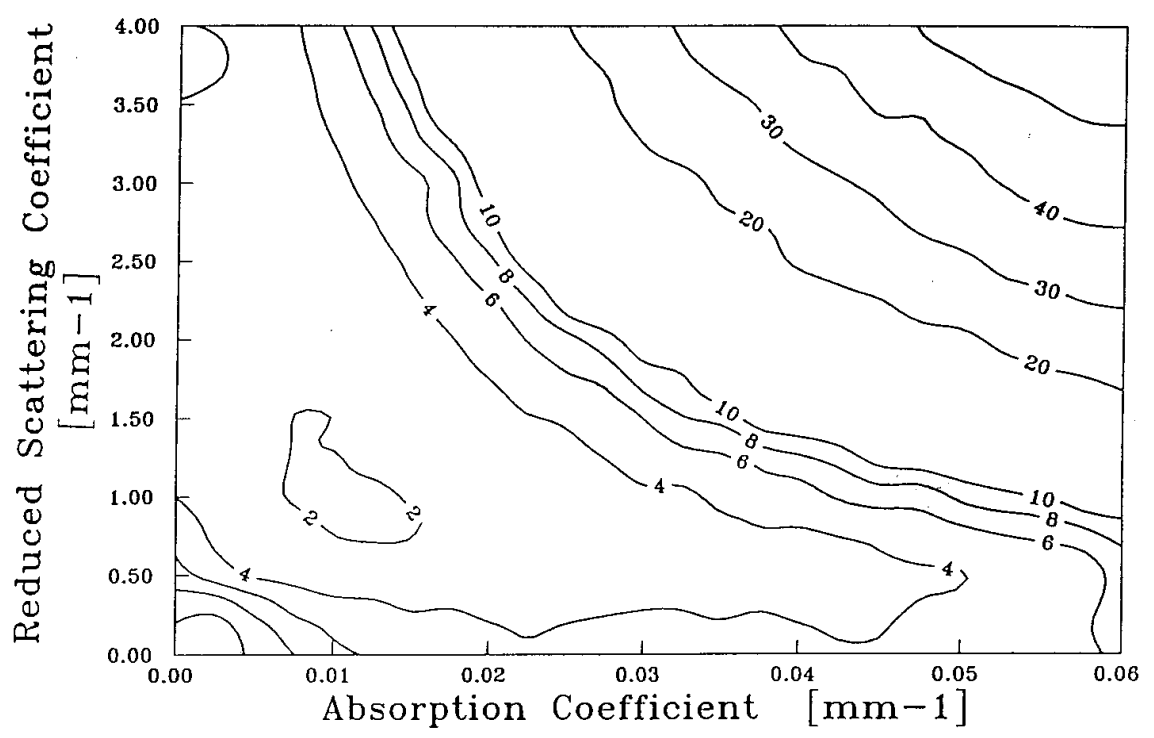

Fig. 6 Calculated percent relative uncertainty in the absorption coefficient under the same conditions as Fig. 5, except that the modulation frequency has been increased to $500 \mathrm{MHz}$.

$\mu_{\mathrm{a}} \mu \mathrm{a}=0.005,0.010$, and $0.020 \mathrm{~mm}^{-1}$. Similar results are shown in Figure 9 where $\mu_{\mathrm{a}}=0.01 \mathrm{~mm}^{-1}$ and $\mu_{\mathrm{s}}^{\prime}=0.5,1.0$, and $2.0 \mathrm{~mm}^{-1}$.

The influence of a curved surface was examined by considering cylindrical and spherical tissue volumes for forward data generation. The analytical solutions of Moulton ${ }^{19}$ for an infinite cylinder and a sphere were used to calculate the reflectance at distances of 1 and $2 \mathrm{~cm}$ along the surface of the object, and these results were used to compute phase difference and intensity ratios. As described above, these data were then used as input to the algorithm based on Eq. (1) and the errors in $\mu_{\mathrm{a}}$ and $\mu_{\mathrm{s}}^{\prime}$ were calculated. These are plotted as a function of cylinder diameter in Figure 10 for $\mu_{\mathrm{s}}^{\prime}=1.0 \mathrm{~mm}^{-1}$ and $\mu_{\mathrm{a}}=0.005,0.010$, and $0.020 \mathrm{~mm}^{-1}$. Results for the spherical case are shown in Figure 11. These absolute errors in tissue optical properties demonstrate the magnitude of systematic errors which would be anticipated in trying to use the semi-infinite medium diffusion theory expression along with real data measured from tissues that have a natural curvature, such as an arm, leg, or head.

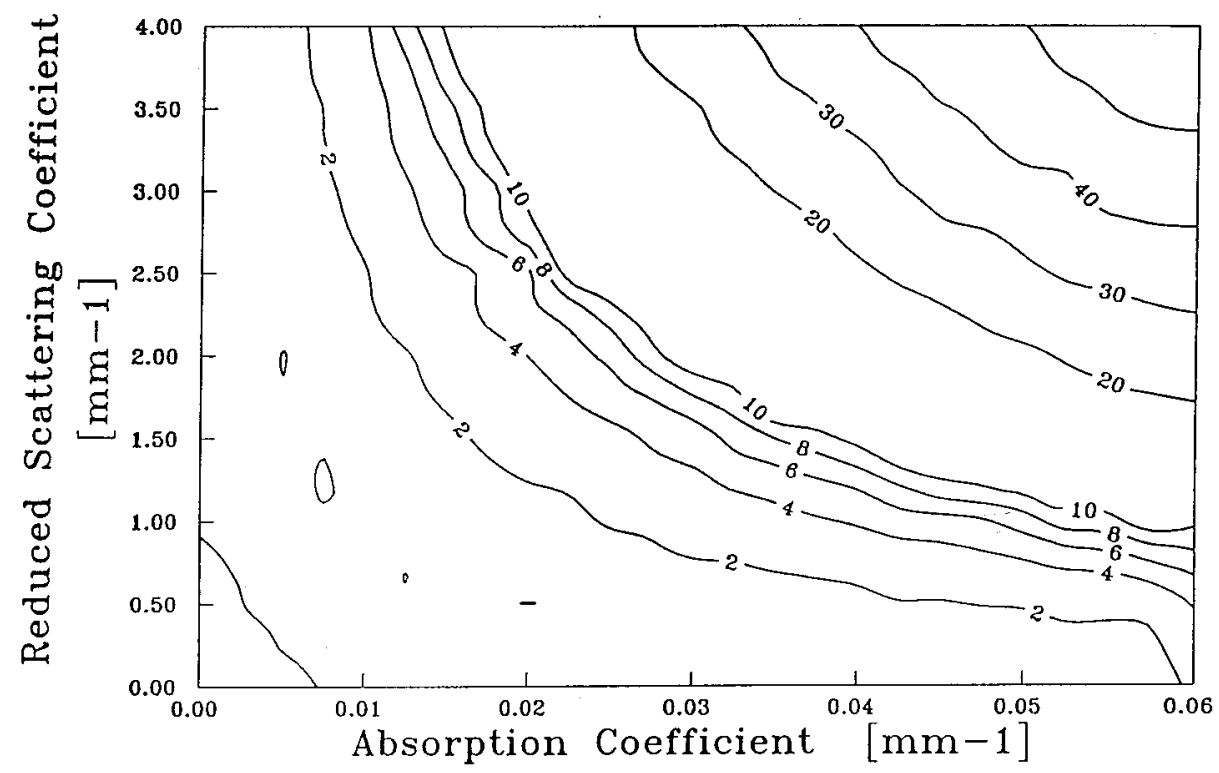

Fig. 7 Calculated percent relative uncertainty in the absorption coefficient under the same conditions as Fig. 5, except that the source modulation has been increased from 8 to $100 \%$. 


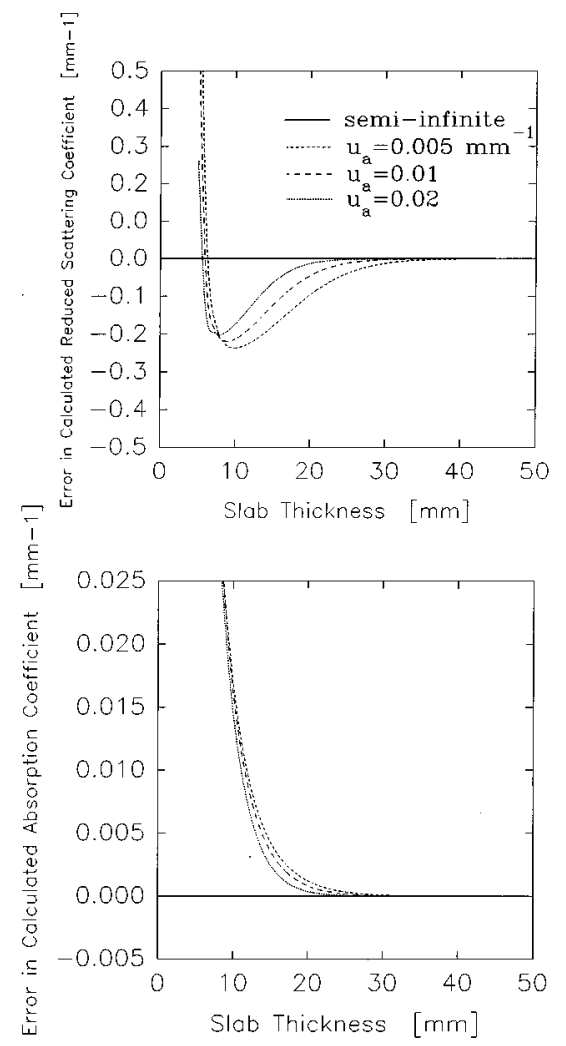

Fig. 8 Calculated absolute errors in the reduced scattering and absorption coefficients as functions of slab thickness for $\mu_{\mathrm{s}}^{\prime}=1.0$ $\mathrm{mm}^{-1}$ and $\mu_{\mathrm{a}}=0.005,0.010$, and $0.020 \mathrm{~mm}^{-1}$. Calculations are for a two-detector system (1 and $2 \mathrm{~cm}$ ) based on phase difference and intensity ratio operated at $135 \mathrm{MHz}$. The systematic error shown here is caused by the assumption of semi-infinite geometry.

For the simplest case, where the tissue volume was considered to be an infinite slab, the effect of using the semi-infinite model in the analysis is an overestimation of $\mu_{\mathrm{a}}$. The reduced scattering coefficient is underestimated, except for very thin slabs. As shown in Figure 8, the absolute error in $\mu_{\mathrm{a}}$ is only weakly dependent on $\mu_{\mathrm{a}}$. As we showed in a previous paper, this suggests that changes in $\mu_{\mathrm{a}}$ can be accurately measured even if the tissue geometry is not well known and not accounted for because all measurements would share a common offset. For the arrangement considered here (i.e., detectors at 1 and $2 \mathrm{~cm}$ ), errors in $\mu_{\mathrm{a}}$ exceed $10 \%$ only when the slab thickness is less than $23 \mathrm{~mm}$ for $\mu_{\mathrm{s}}^{\prime}=1.0$ $\mathrm{mm}^{-1}$. For lower values of $\mu_{\mathrm{s}}^{\prime}$ (see Figure 9) this occurs at a somewhat greater thickness, but a practical rule of thumb is to use the semi-infinite model with caution if the tissue thickness is less than the maximum source-detector separation. This rule assumes that the optical properties are within the representative range examined here.

The results for cylindrical and spherical tissue volumes are shown in Figures 10 and 11, respectively. In each case both $\mu_{\mathrm{a}}$ and $\mu_{\mathrm{s}}^{\prime}$ are underestimated for objects with diameters between 20 and

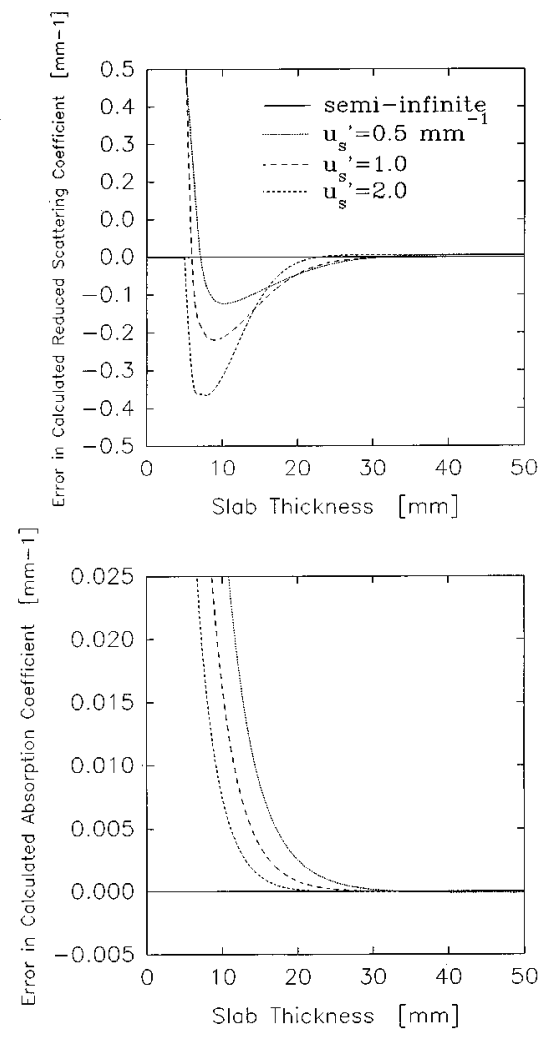

Fig. 9 Similar to Fig. 8 for the case of $\mu_{\mathrm{a}}=0.01 \mathrm{~mm}^{-1}$ and $\mu_{\mathrm{s}}^{\prime}$ $=0.5,1.0$, and $2.0 \mathrm{~mm}^{-1}$.

$150 \mathrm{~mm}$. This is in contrast to our previous results for a one-detector (i.e., phase and modulationbased) system where $\mu_{\mathrm{s}}^{\prime}$ was underestimated and $\mu_{\mathrm{a}}$ was overestimated. The magnitude of the errors is comparable with either approach, although the two-detector system is somewhat inferior overall. The finite-volume studies were disappointing because we had initially hoped that a two-detector system would be less prone to these sorts of errors. This is not the case, however, and the semi-infinite model must be used with caution, especially if the goal is to recover the absolute absorption and/or scattering coefficient. Of course more complex models can be used if sufficient information is available about the tissue geometry, but this is often not the case in biomedical applications.

\subsection{RESULTS OF IN VIVO MEASUREMENTS}

In Figure 12 we show the absorption and scattering spectra for two of the rabbits measured before the injection of any $\mathrm{NiPcS}_{4}$ and after injection of the last aliquot (total doses of $\mathrm{NiPcS}_{4}, 7.6 \mathrm{mg} \mathrm{kg}^{-1}$ and 20.3 $\left.\mathrm{mg} \mathrm{kg}^{-1}\right)$. The representative error bars show the standard deviations in $\mu_{\mathrm{a}}$ and $\mu_{\mathrm{s}}^{\prime}$ based on the four measurements of phase and intensity at each source-detector distance. The absorption and scat- 


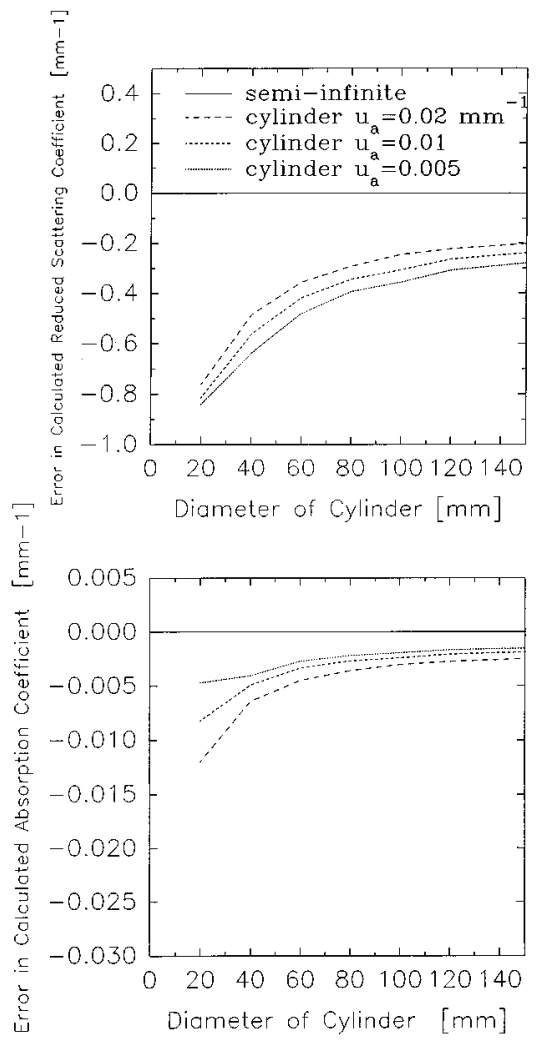

Fig. 10 Calculated systematic errors in $\mu_{\mathrm{a}}$ for cylindrical tissue volumes with $\mu_{\mathrm{s}}^{\prime}=1.0 \mathrm{~mm}^{-1}$ and $\mu_{\mathrm{a}}=0.005,0.010$, and 0.020 $\mathrm{mm}^{-1}$.

tering coefficients at $660 \mathrm{~nm}$ are plotted as functions of the total injected dose of $\mathrm{NiPcS}_{4}$ for all three rabbits in Figure 13.

These measurements show how the frequency domain system can be used to address a biological problem: measurement of the uptake kinetics of a dye in a specific organ. While there was considerable variation in the absolute values of $\mu_{\mathrm{a}}$ and $\mu_{\mathrm{s}}^{\prime}$ for the muscle itself, the increase in $\mu_{\mathrm{a}}$ for a given injected dose of $\mathrm{NiPcS}_{4}$ was similar for all three animals. The increase also depended linearly on total injected dose, which would be expected since the dye remains in circulation for the duration of the experiment. From measurements of the extinction coefficient of $\mathrm{NiPcS}_{4}$ in a conventional spectrometer, we calculated the average concentration of the dye in the muscle and the specific uptake ratio (i.e., ratio of muscle concentration to injected dose). This was $0.104,0.134$, and 0.135 for rabbits 1,2 , and 3 respectively. Assuming the total body blood volume to be $60 \mathrm{ml} / \mathrm{kg}^{27}$ and the average concentration of blood in the muscle to be $1 \%$, the expected uptake ratio would be 0.167 , so the results are quite reasonable. No significant change was seen in $\mu_{s}^{\prime}$ in any of the animals, demonstrating the ability of this approach to measure changes in absorption in the presence of multiple scattering.

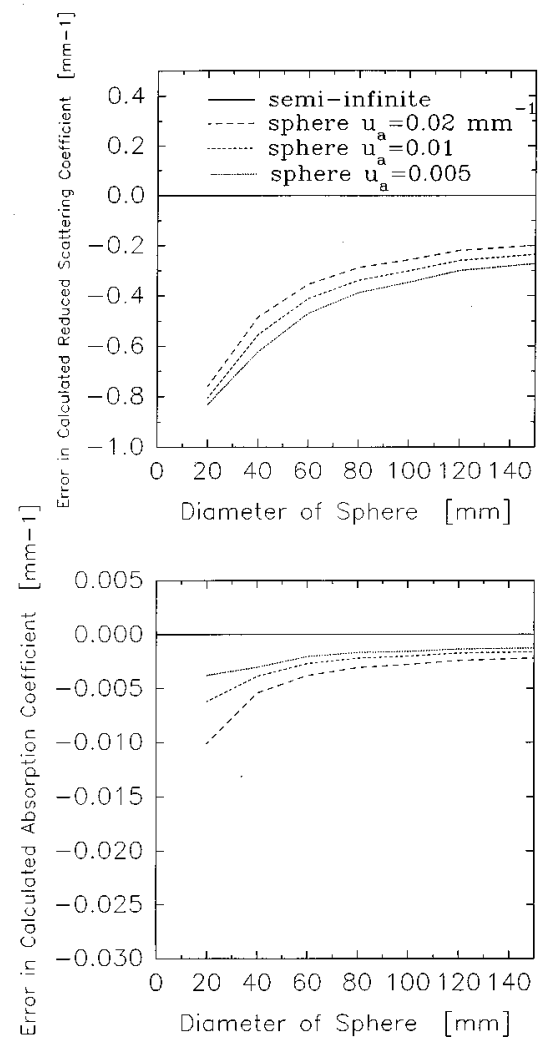

Fig. 11 Calculated systematic errors in $\mu_{\mathrm{a}}$ and $\mu_{\mathrm{s}}^{\prime}$ for spherical tissue volumes with $\mu_{\mathrm{s}}^{\prime}=1.0 \mathrm{~mm}^{-1}$ and $\mu_{\mathrm{a}}=0.005,0.010$, and $0.020 \mathrm{~mm}^{-1}$.

The values of $\mu_{\mathrm{s}}^{\prime}$ and $\mu_{\mathrm{a}}$ are both low compared with literature values for skeletal muscle, but it should be noted that there are few in vivo measurements for muscle at all, and none that we are aware of for the rabbit. As discussed earlier, these absolute values could be subject to significant systematic error caused by the finite tissue volume. Because we pressed a plate onto the surface of the muscle, the measurement geometry more closely resembled a slab than a sphere or cylinder. Under these conditions we would expect the scattering coefficient to be underestimated (see Figures 8 and 9) and the absorption coefficient to be overestimated. However, it is unlikely that $\mu_{\mathrm{a}}$ could actually be lower than the measured value, because if the blood content is $1 \%$ and the hemoglobin oxygenation is $75 \%, \mu_{\mathrm{a}}$ should be about $0.008 \mathrm{~mm}^{-1}$ at 660 $\mathrm{nm}$ due to the presence of blood alone. ${ }^{28}$ As discussed earlier, changes in $\mu_{\mathrm{a}}$ can be determined more accurately and we believe the uptake measurements to be reliable.

\section{CONCLUSIONS}

The first goal of this paper was to characterize the uncertainty in phase and intensity and to examine how these errors propagate into estimates of $\mu_{\mathrm{a}}$ and 

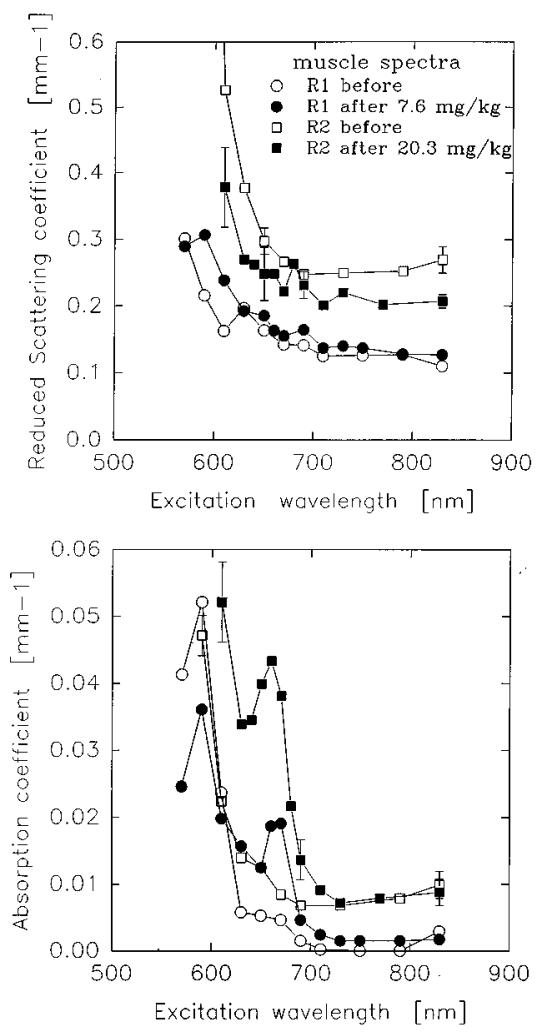

Fig. 12 Reduced scattering and absorption spectra measured on the femoral biceps muscle of two rabbits before (hollow symbols) and after (solid symbols) the injection of $\mathrm{NiPcS}_{4}$. Rabbit 1 (circles) was injected with a total dose of $7.6 \mathrm{mg} \mathrm{kg}^{-1}$ and rabbit 2 (squares) received a total dose of $20.3 \mathrm{mg} \mathrm{kg}^{-}$

$\mu_{\mathrm{s}}^{\prime}$. We found that for typical signal levels, the noise in our system is dominated by photon shot noise. The effects of this noise can be minimized by using a two-detector system based on measurements of intensity ratio and phase difference rather than a one-detector system that relies on measurements of phase and modulation relative to the source. For best results, signals from the two detectors should be equalized so that photon noise in the weaker signal is not dominant. We demonstrated that increasing the modulation depth and frequency is also advantageous, although not practical with the arc lamp source. Our system can achieve, at best, about $4 \%$ uncertainty in $\mu_{\mathrm{a}}$ and $\mu_{\mathrm{s}}^{\prime}$ if the signals at the two detectors are equalized (Figure 5). As also found by Fantini et al., ${ }^{14}$ this could be reduced to about $1 \%$ by using a source with $100 \%$ modulation. Whether this accuracy is adequate will depend on the demands of the specific biomedical application to which these techniques are applied.

Systematic errors due to finite tissue volumes and curved surfaces can be much larger than random errors due to noise. These systematic errors can presumably be reduced if enough information about the tissue geometry is available to justify the use of a more correct model. Such models are, however,
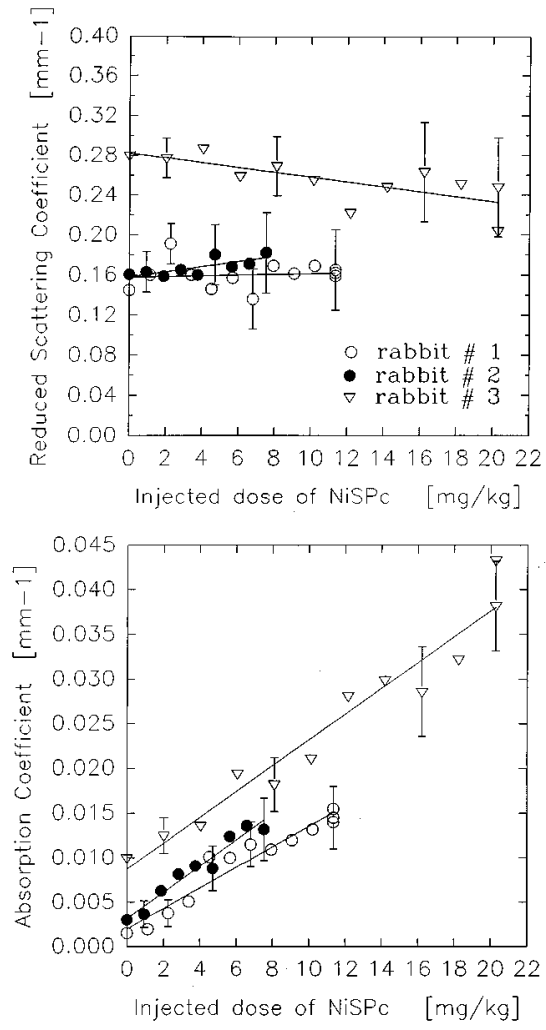

Fig. 13 Reduced scattering and absorption coefficients at $660 \mathrm{~nm}$ measured for three rabbits as functions of increasing total injected dose of $\mathrm{NiPCS}_{4}$.

more complex to implement since they rely on the calculation of an infinite series.

Measurements made in vivo suggest that it is difficult to achieve the shot noise limits in practice. From the in vivo data presented in Figures 12 and 13 , the standard deviation in $\mu_{\mathrm{a}}$ and $\mu_{\mathrm{s}}^{\prime}$ is typically $20 \%$ for a 15 s measurement time. Referring to Fig. 4 , the expected errors due to shot noise for $\mu_{\mathrm{s}}^{\prime}$ $=0.2, \mu_{\mathrm{a}}=0.1$ would be $30 \%$ in $\mu_{\mathrm{a}}$ and $15 \%$ in $\mu_{\mathrm{s}}$ for a $1 \mathrm{~s}$ data acquisition. Hence the shot-noise contribution to the noise for a $15 \mathrm{~s}$ measurement should be only about $8 \%$ in $\mu_{\mathrm{a}}$ and $4 \%$ in $\mu_{\mathrm{s}}^{\prime}$. This suggests that other sources of variation such as movement or changes in tissue blood content or oxygenation with time were more important than the inherent instrument noise in determining the precision of the $\mu_{\mathrm{a}}$ and $\mu_{\mathrm{s}}^{\prime}$ estimates.

There are other possible sources of error that have not been considered in this paper. Boas et al. ${ }^{29}$ have described how uncertainty in source-detector position can be the dominant source of error in optical tomography. In our case, the source and detector are fixed in a jig and the tissue is regarded as homogeneous, so that displacement of the source and detector together should not affect the signals. In practice, and as noted for the in vivo case, tissue is not homogeneous so that a change in the relative 
location of an inhomogeneity such as a blood vessel could affect the measurements.

We have also disregarded the effects of finite source and detector size as well the details of their angular dependence. Previous examinations of these factors ${ }^{30,31}$ suggest that at the relatively large source-detector distances used in this study they are not a source of significant error. A more fundamental question is the validity of the simple diffusion model. A preliminary comparison with Monte Carlo simulations as well as the results of Fantini et al. ${ }^{21}$ suggests that significant errors in $\mu_{\mathrm{a}}$ and $\mu_{\mathrm{s}}$ ( 5 to $10 \%$ ) can be attributed to the diffusion model under worst-case conditions. We plan to examine this more closely in the future, but even if a more correct semi-infinite model is used, the errors due to measurement noise and finite volumes remain. Of course a semi-infinite homogeneous model ignores the real structure of the tissue and highly absorbing inhomogeneities such as blood vessels. Whether useful physiological information can be obtained under such simple assumptions remains to be seen, but the in vivo experiments reported here suggest that it can.

\section{Acknowledgments}

This work was supported by the National Institutes of Health Grant PO1-CA 43892. The authors are grateful to Joe Hayward for his assistance with the animal experiments, Alwin Kienle for the use of his Monte Carlo data results, and to Donna Laking for her preparation of the manuscript.

\section{REFERENCES}

1. W. F. Cheong, S. A. Prahl, and A. J. Welch, "A review of the optical properties of biological tissues," IEEE J. Quantum Elect. 26, 2166-2185 (1990).

2. R. A. J. Groenhuis, H. A. Ferwerda, and J. J. ten Bosch, "Scattering and absorption of turbid materials determined from reflection measurements. 1: Theory," Appl. Opt. 22, 2456-2462 (1983).

3. T. J. Farrell, M. S. Patterson, and B. C. Wilson, "A diffusion theory model of spatially resolved, steady-state diffuse reflectance for the noninvasive determination of tissue optical properties in vivo," Med. Phys. 19, 879-888 (1992).

4. S. L. Jacques, A. Gutsche, J. Schwartz, L. Wang and F. K. Tittel, "Video reflectometry to extract optical properties of tissue in vivo," in Medical Optical Tomography: Functional Imaging and Monitoring, G. Muller, Ed. pp. 211-226, SPIE Optical Engineering Press, Bellingham (1993).

5. R. Splinter, G. A. Nanney, L. Littman, C. H. Chuang, R. H. Svenson, J. R. Tuntelder and G. P. Tatsis, "Monitoring tissue optical characteristics in situ using a CCD camera," Lasers Life Sci. 6, 15-25 (1994).

6. R. A. Bolt and J. J. ten Bosch, "On the determination of optical parameters for turbid materials," Waves in Random Media 4, 233-242 (1994).

7. D. T. Delpy, M. Cope, P. van der Zee, S. Arridge, S. Wray, and J. Wyatt, "Estimation of optical pathlength through tissue from direct time-of-flight measurements," Phys. Med. Biol. 33, 1433-1442 (1988).

8. M. S. Patterson, B. Chance, and B. C. Wilson, "Timeresolved reflectance and transmittance for the noninvasive measurement of tissue optical properties," Appl. Opt. 28, 2331-2336 (1989)

9. M. Ferrari, Q. Wei, L. Carraresi, R. A. deBlasi, and G.
Zacuanti, "Time-resolved spectroscopy of the human forearm," J. Photochem. Photobiol. B: Biol. 16, 141-153 (1992).

10. H. Liu, M. Miwa, B. Beauvoit, N. G. Wang, and B. Chance, "Characterization of absorption and scattering properties of small-volume biological samples using time-resolved spectroscopy," Anal. Biochem. 213, 378-385 (1993).

11. M. S. Patterson, J. D. Moulton, B. C. Wilson, K. W. Berndt, and J. R. Lakowicz, "Frequency-domain reflectance for the determination of the scattering and absorption properties of tissue," Appl. Opt. 30, 4474-4476 (1991).

12. S. J. Madsen, P. Wyss, L. O. Svaasand, R. C. Haskell, Y. Tadir, and B. J. Tromberg, "Determination of the optical properties of the human uterus using frequency-domain photon migration and steady state techniques," Phys. Med. Biol. 39, 1191-1202 (1994).

13. S. J. Madsen, E. R. Anderson, R. C. Haskell, and B. J. Tromberg, "Portable high-bandwidth frequency-domain photon migration instrument for tissue spectroscopy," Opt. Lett. 19, 1934-1936 (1994).

14. S. Fantini, M. A. Franceschini-Fantini, J. S. Maier, S. A. Walker, B. Barbieri, and E. Gratton, "Frequency-domain multichannel optical detector for noninvasive tissue spectroscopy and oximetry," Opt. Eng. 34, 32-42 (1995).

15. E. M. Sevick, B. Chance, J. Leigh, S. Nioka, and M. Maris, "Quantitation of time- and frequency-resolved optical spectra for the determination of tissue oxygenation," Anal. Biochem. 195, 330-351 (1991).

16. M. S. Patterson, "Noninvasive measurement of tissue optical properties: current status and future prospects," Comments Mol. Cell Biophys. 8, 387-417 (1995).

17. B. W. Pogue and M. S. Patterson, "Frequency-domain optical absorption spectroscopy of finite tissue volumes using diffusion theory," Phys. Med. Biol. 39, 1157-1180 (1994).

18. M. S. Patterson, B. C. Wilson, and D. R. Wyman, "The propagation of optical radiation in tissue I. Models of radiation transport and their application," Lasers Med. Sci. 6, 155168 (1991)

19. J. D. Moulton, "Diffusion Modelling of Picosecond Laser Pulse Propagation in Turbid Media," M. Eng. thesis, McMaster University, Hamilton, Canada (1990).

20. S. R. Arridge, M. Cope, and D. T. Delpy, "The theoretical basis for the determination of optical pathlengths in tissue: temporal and frequency analysis," Phys. Med. Biol. 37, 15311560 (1992).

21. S. Fantini, M. A. Franceschini, and E. Gratton, "Semiinfinite-geometry boundary problem for light migration in highly scattering media: a frequency-domain study in the diffusion approximation," J. Opt. Soc. Am. B 11, 2128-2138 (1994).

22. R. C. Haskell, L. O. Svaasand, T. T. Tsay, T. C. Feng, M. S. McAdams, and B. J. Tromberg, "Boundary conditions for the diffusion equation in radiative transfer," J. Opt. Soc. Am. A 11, 2727-2741 (1994).

23. F. P. Bolin, L. E. Preuss, R. C. Taylor, and R. J. Ference, "Refractive index of some mammalian tissues using a fiber optic cladding method," Appl. Opt. 28, 2297-2303 (1989).

24. S. Fantini, M. A. Franceschini, J. B. Fishkin, B. Barbieri, and E. Gratton, "Quantitative determination of the absorption spectra of chromophores in strongly scattering media: a light-emitting-diode based technique," Appl. Opt. 33, 52045213 (1994).

25. E. Gratton and M. Linkeman, "A continuously variable frequency cross-correlation phase fluorometer with picosecond resolution," Biophys. J. 44, 315-324 (1983).

26. A. B. Carlson, Communication Systems: An Introduction to Signals and Noise in Electrical Communication, McGraw Hill, New York (1986).

27. E. D. Olfert, B. M. Cross, and R. A. McWilliam, Eds., Guide to the Care and Use of Experimental Animals Vol. 1, Canadian Council on Animal Care, Bradda, Ottawa, Canada (1993).

28. M. Cope and D. T. Delpy, "System for long-term measurement of cerebral blood and tissue oxygenation on newborn infants by near infra-red transillumination," Med. Biol. Eng. Comput. 26, 289-294 (1988).

29. D. A. Boas, M. A. O'Leary, B. Chance, and A. G. Yodh, "Practical limits for the detection and characterization of 
optical inhomogeneities using diffuse photon density waves," Technical Digest, Optical Society of America Meeting on Advances in Optical Imaging and Photon Migration, pp. 61-63 (1996).

30. A. Kienle, L. Lilge, M. S. Patterson, R. Hibst, R. Steiner, and B. C. Wilson, "Spatially resolved absolute diffuse reflectance measurements for non-invasive determination of the optical scattering and absorption coefficients of biological tissue," Appl. Opt. 35, 2304-2314 (1996).

31. S. J. Madsen, B. C. Wilson, M. S. Patterson, Y. D. Park, S. C. Jacques, and Y. Hefetz, "Experimental tests of a simple diffusion model for the estimation of scattering and absorption coefficients of turbid media from time-resolved diffuse reflectance measurements," Appl. Opt. 31, 3509-3517 (1992). 